\title{
Myotubularin family phosphatase ceMTM3 is required for muscle maintenance by preventing excessive autophagy in Caenorhabditis elegans
}

\author{
Xiaokun $\mathrm{Yu}^{1,2}$, Junfeng $\mathrm{Ma}^{1,2}$, Feng Lin ${ }^{1,2}$, Wanke Zhao ${ }^{1}$, Xueqi $\mathrm{Fu}^{2}$ and Zhizhuang Joe Zhao ${ }^{{ }^{*}}$
}

\begin{abstract}
Background: Autophagy is a ubiquitous cellular process responsible for the bulk degradation of cytoplasmic components through the autophagosomal-lysosomal pathway. In skeletal muscle, autophagy has been regarded as a key regulator for muscle mass maintenance, and its imbalance leads to sarcopenia. However, the underlying mechanism is poorly understood.

Results: In this study, we demonstrate that ceMTM3, a FYVE-domain containing myotubalarin family phosphatase, is required for the maintenance of muscle fibers by preventing excessive autophagy in Caenorhabditis elegans. Knockdown of ceMTM3 by using feeding-based RNA interference caused loss of muscle fibers accompanied by shortening of muscle cell and body size in aged C. elegans worms. This was preceded by the occurrence of excessive autophagy in the muscle and other tissues, which subsequently resulted in increased lysosomal activity and necrotic cell death. However, knockdown of ceMTM3 did not aggravate the abnormalities of muscle wasting in autophagy-deficient atg-18 mutant worms.
\end{abstract}

Conclusions: Our data suggest an important role of ceMTM3 in regulating autophagy and maintaining muscle fibers. This study may have clinical implications for prevention and treatment of sarcopenia.

Keywords: Phosphatase, Myotubalarin, RNAi, Autophagy, Muscle, C. elegans, Sarcopenia

\section{Background}

Autophagy is an evolutionarily conserved intracellular process by which cytoplasmic constituents, including long-lived proteins, protein aggregates, organelles, and invading pathogens, are delivered to lysosomes for degradation and subsequent recycling [1]. Autophagy is activated in response to changes in the internal status of the cell and/or changes in the extracellular environment and plays an essential role in cell and tissue homeostasis. Deregulation of autophagy has been linked to many human diseases such as cancer, neurodegeneration, myopathies, diabetes, and infections by bacteria and viruses [2].

Genetic screens, primarily in the yeast $S$. cerevisiae, have identified numerous autophagy-related genes (ATG),

\footnotetext{
* Correspondence: joe-zhao@ouhsc.edu

'Department of Pathology, University of Oklahoma Health Sciences Center, Oklahoma City, Oklahoma 73104, USA

Full list of author information is available at the end of the article
}

many of which have orthologs in higher eukaryotes, including C. elegans, Drosophila, and mammals [1,3]. The products of these genes constitute two unique protein conjugation systems responsible for formation and elongation of autophagic isolation membranes during autophagy [4]. In addition to the conjugation systems, autophagy initiation is also dependent on phosphatidylinositol 3-phosphate (PI3P) based on the crucial role of the type III PI3Kinase Vps34, an enzyme that generates PI3P and forms a complex with autophagy regulator Beclin-1 (ATG-6) [5]. The level of PI3P is also controlled by PI3P phosphatases that belong to the myotubularin family in mammalian cells [6], which presumably play a role in regulation of autophagy as well. Indeed, studies have demonstrated that PI3P phosphatase Jumpy and myotubularin-related phosphatase 3 (MTMR3) act as negative regulators of autophagy in mammalian cells $[7,8]$.

Myotubularin phosphatases are members of the protein tyrosine phosphatase superfamily [6]. But unlike other protein phosphatases, myotubularin family enzymes

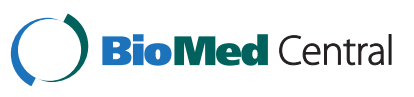


dephosphorylate PI3P and phosphatidylinositol (3, 5)bi-phosphate [6]. Mutations in genes encoding myotubularin proteins are associated with diseases. For example, mutations in MTM1, the founding member of this family, cause X-linked myotubular myopathy (XLMTM), a severe congenital muscular disorder, while mutations in MTMR2 and MTMR13 are associated with Charcot-Marie-Tooth disease [9-11]. We previously have isolated and characterized a C. elegans homolog of myotubularin proteins, designated ceMTM3. ceMTM3 preferably dephosphorylates PI3P and contains a FYVE lipid-binding domain at its C-terminus which binds to PI3P [12]. Knockdown of ceMTM3 in $C$. elegans worms by using feeding-based RNA interference caused severe impairment of body movement following post-reproductive age and also significantly shortened their lifespan [12]. We reasoned that this may be related to loss of muscle function due to de-regulation of autophagy. In this study, we demonstrate that knockdown of ceMTM3 induces autophagy that precedes an accelerated loss of muscle fibers in C. elegans worms.

\section{Results and discussion}

Knockdown of ceMTM3 causes loss of muscle fibers in adult C. elegans

In an earlier study, we isolated and characterized a C. elegans homolog of myotubularin phosphatases, designated ceMTM3 [12]. ceMTM3 is predominately expressed in muscle of adult $C$. elegans worms. It binds PI3P through its C-terminal FYVE domain and preferably dephosphorylates PI3P. Knockdown of ceMTM3 by using feeding-based RNA interference leads to near total loss of ceMTM3 expression and causes a gradual impairment of body movement from day 5 with significant shortening of lifespan of the worms [12]. Since ceMTM3 is predominantly expressed in the muscle [12], the progressive locomotory impairment associated with knockdown of the enzyme may be caused by declining muscle functions. To verify this, we employed Alexa Fluor 568-conjugated phalloidin to detect actin fibers in whole-mount worms (Figure 1A). On day 3, both control and RNAi-treated worms displayed clear and organized actin fibers. However, on day 5 , clear deterioration of the fibers was seen with the RNAi-treated worms, and by day 9 the fibers were essentially absent, which correlated with the total impairment of body movement. In contrast, the control worms still maintained actin fiber structure on day 9, although not as organized as that seen with younger worms. By day 15 , control worms also displayed significant loss of muscle fibers. Loss of muscle fibers is a progressive event as the worm ages, but knockdown of ceMTM3 markedly accelerates the process. Therefore, our data indicate that ceMTM3 is required to stabilize muscle fibers in adult $C$. elegans worms. To further verify the effects of ceMTM3 knockdown on muscle fibers, we employed RW1596 worms which express GFP:: MHC A carried by a construct in which a GFP coding sequence was inserted at the translation initiation codon in the gene for myosin heavy chain A [13]. The data are shown in Figure 1B. On day 3 , both control and RNAi-treated young adult worms showed strong and organized muscle fibers. However, on day 5, while the muscle fibers in control worms displayed a slight decrease, those in the ceMTM RNAitreated worms were markedly reduced. Quantification of GFP fluorescence signals revealed near 50\% loss of GFPmyosin in the treated worms on day 5 . The data provide further evidence that knockdown of ceMTM3 destabilize muscle fibers which contain both myosin and actin.

\section{Knockdown of ceMTM3 shortens the body size of adult C. elegans}

Besides the impairment of locomotion, knockdown of ceMTM3 also affected the body size of $C$. elegans worms. As shown in Figure 2A and B, control C. elegans entered reproductive period on day 3 , but they continued to grow until around day 5 to day 7. However, ceMTM3 RNAi-treated worms started to shrink after day 3. On day 7 , the average size of ceMTM3 RNAitreated worms were $8-15 \%$ shorter than the control worms of the same age. The shortening of body length is apparently due to a reduction in the size of individual muscle cells as revealed by staining of muscle actin fibers with Alexa Fluor 568-conjugated phalloidin (Figure 2C). On average, the muscle cell size was reduced by about $30 \%$ (Figure 2D). It should be noted that knockdown of ceMTM3 had no significant effects on the development of worms from embryos to young adults (data not shown).

\section{Knockdown of ceMTM3 causes occurrence of autophagy in multiple cells of adult $C$. elegans worms}

We hypothesized that the loss of muscle fibers may be caused by death of muscle cells due to autophagy, which is known to have an important role in muscle maintenance and to be dependent on PI3P [5,14]. Our earlier studies have shown the knockdown of ceMTM3 affects the total level of PI3P in C. elegans worms [12]. It is likely that loss of ceMTM function causes autophagy, thereby leading to loss of muscle fibers. To visualize autophagy, we used worms carrying a GFP::LGG-1 fusion protein. LGG-1 is an ortholog of yeast Atg8p and mammalian MAP-LC3 in C. elegans. During autophagy, GFP-labeled LGG-1 protein localizes to preautophagosomal and autophagosomal membranes and forms green puncta, thereby serving as a reporter for autophagy [15]. Data shown in Figure 3 demonstrate occurrence of autophagy in multiple cells in ceMTM3 RNAi-treated 


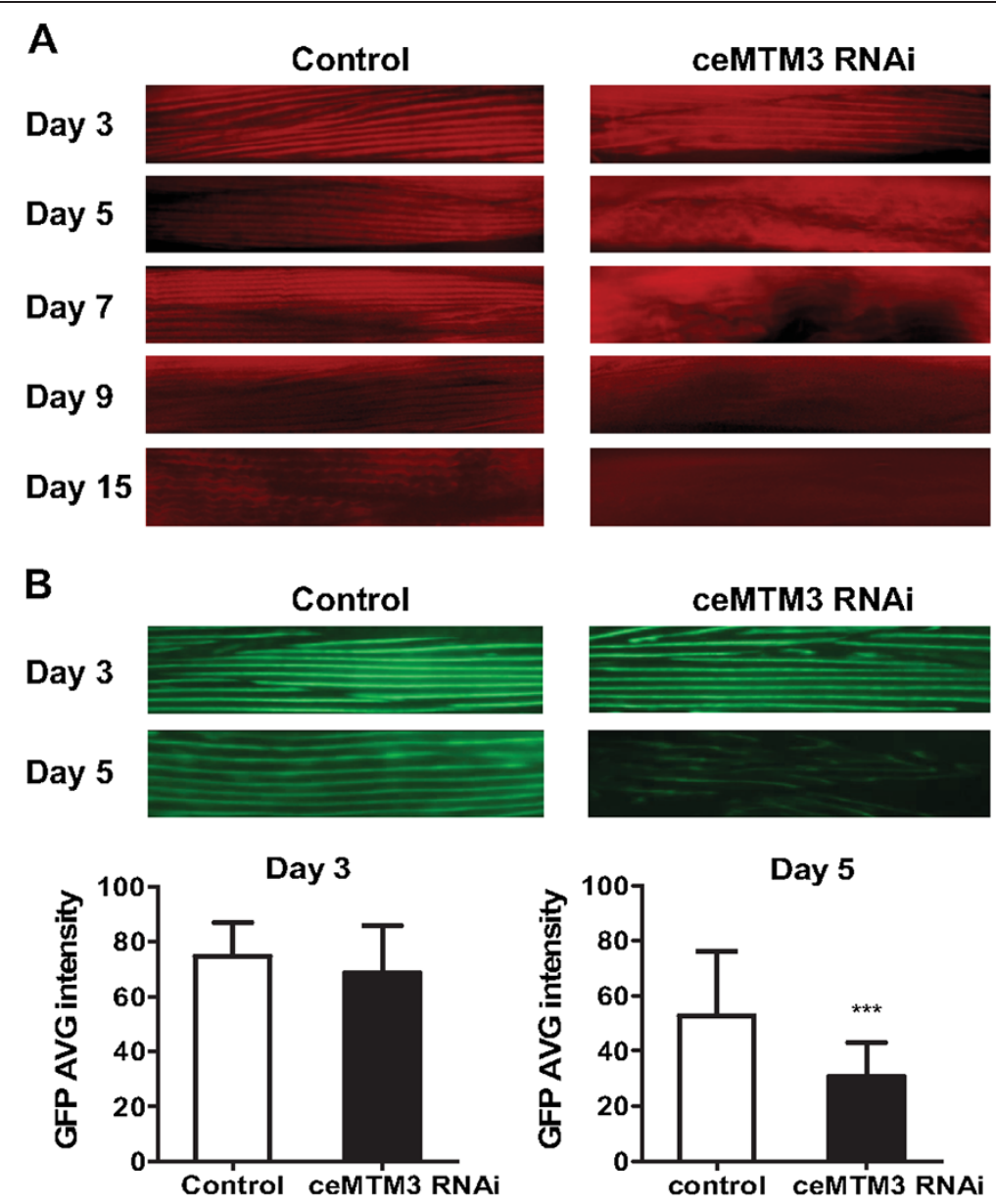

Figure 1 Knockdown of ceMTM3 causes loss of muscle fibers in adult C. elegans. Normal N2 and RW1596 worms were cultured on NGM plates containing E. coli cells carrying vector control or ceMTM3 RNAi from the time they were hatched from eggs. A. Alexa Fluor 568- phalloidin staining of muscle fibers in normal N2 worms at indicated ages. B. Images of GFP-positive muscle fibers and quantification of GFP intensity in day 3 and day 5 transgenic RW1596 worms which express a GFP::myosin heavy chain A fusion protein. Data represents mean \pm SD $(n=50)$. *** $p<0.001$.

worms on different days. Autophagy occurred in seam cells in day 2 larvae, in muscle cells in day 3 young adults, and day 4 and thereafter in intestine of adult worms (Figure 3A-D). In the control worm, such structures were essentially absent until day 12 when a few sporadic punctuates appeared in the intestine (not shown). We also confirmed induction of autophagy by detecting lipidation of GFP-LGG-1, another hallmark of autophagy. As shown in Figure 3E, upon treatment of C. elegans worms with ceMTM3 RNAi, a clear band of $\sim 37 \mathrm{kDa}$ was recognized by anti-GFP antibody in the membrane extracts of worms. Together, our data indicate that knockdown of ceMTM3 caused a marked increase in autophagy activity. It is worth noting that the onset of autophagy apparently preceded the deterioration of muscle fibers which began on day 5 (see Figure 1). Apparently, the presence of ceMTM3 prevents occurrence of autophagy in the normal worms as they age. The occurrence of autophagy in muscle and intestine is consistent with the distributions of the enzyme in these tissues as revealed in our earlier studies [12].

\section{Knockdown ceMTM3 increases lysosomal activities and} induces necrotic cell death in C. elegans

After formation, autophagosomes deliver the sequestered cytoplasmic materials into lysosomes for further degradation [1]. To find out whether the formation of autophagosomes induced by knockdown of ceMTM3 has further functional consequences, we examined the lysosomal activities by using acridine orange, which is commonly used for staining of lysosomes [16,17]. The results showed that knockdown of ceMTM3 induced appearance of numerous lysosomal vesicles in the head region of day 4 adult worms (Figure 4). These vesicles were apparently in muscle cells as the muscle fibers were visible in DIC images. The lysosomal vesicles were also 

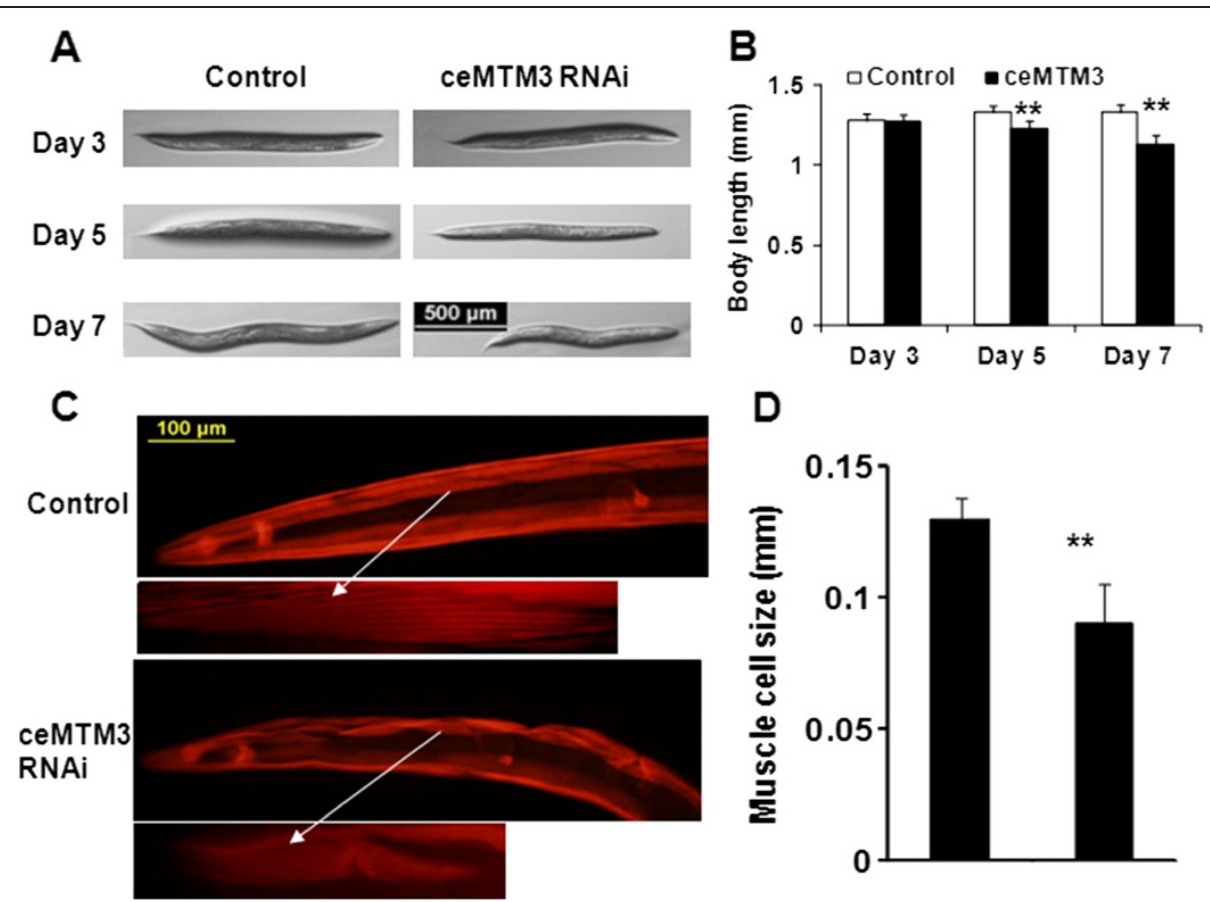

Figure 2 ceMTM3 knockdown-induced loss of muscle fibers is accompanied by shortening of body size in aged C. elegans worms.

Normal N2 worms were cultured on NGM plates containing E. coli cells carrying vector control or ceMTM3 RNAi from the time they were hatched from eggs. A. Photography of typical worms at day 3, day 5, and day 7. B. Statistical comparison of body length of worms. Data represents mean \pm SD $(n \geq 12) .{ }^{* *} p<0.01$. C. Revelation of muscle cells and muscle fibers by Alexa Fluor 568 phalloidin staining of day 5 worms. D. Statistical comparison of muscle size of worms. Data represents mean \pm SD ( $n \geq 14)$. ${ }^{* *} p<0.001$.

seen in the body wall muscle although less predominant (not shown). Excessive lysosomes induce cytoplasmic acidification and further lead to necrotic cell death. In fact, studies have demonstrated that autophagy is required for necrotic cell death in C. elegans [18]. As expected, knockdown of ceMTM3 caused significant necrosis as shown in Figure 5. In the head region of ceMTM3 RNAi-treated worms, necrotic cell death, as indicated by cell corpses, started to appear on day 5 and became overwhelming by day 7 . However, this was not seen in control worms, although sporadic cell necrotic cell death was also occasionally observed on day 7 and thereafter. On average, control day 7 worms displayed $0.3 \pm 0.2$ corpses in the head region while ceMTM3 RNAi-treated worms of the same age showed $4 \pm 0.6$ corpses $(\mathrm{p}<0.001)$. Necrotic cell death was also seen in the body wall muscle of RNAi-treated worms on day 7 (not shown).

\section{Knockdown ceMTM3 does not aggravate the} abnormalities of muscle wasting in autophagy-deficient worms

The above data suggest knockdown of ceMTM3 function leads to autophagy which in turn causes cell death and loss of muscle fibers. To further address the causal relationship between loss of muscle fibers and onset of excessive autophagy, we employed autophagy deficient VC893 worms. These worms lack ATP-18, an ortholog of yeast Atg18p and human WIPI, and thus are not able to recruit autophagic membranes to form autophagosomes $[18,19]$. Interestingly, VC893 worms fed on normal $E$. coli food exhibited impaired locomotion after day 5 (Figure 6A). This was associated with deterioration of muscle fibers as revealed by Alexa Fluor 568-conjugated phalloidin staining (Figure 6B, see also Figure 1A for comparison). Nonetheless, knockdown of ceMTM3 did not further exacerbate this abnormality, implying that induction of autophagy is required for ceMTM3 RNAiinduced muscle fiber loss.

The data described above demonstrated that knockdown of a MTM family enzyme causes autophagy and subsequently causes loss of muscle fibers. As a PI3P phosphatase, ceMTM3 should play an important role in controlling the level of PI3P. The involvement of PI3P in autophagy has been demonstrated for the most part by studying the type III PI3Kinase Vps34. Vps34 is a type III PI3 kinase responsible for generation of PI3P. It forms a complex with its autophagy-regulatory partner Beclin-1 (ATG-6), thereby initiating autophagy [5]. Vps34 also has an essential role in membrane trafficking and endocytosis, and Vps34 mutant worms display lethality and molting defects as well as alterations in the 
A

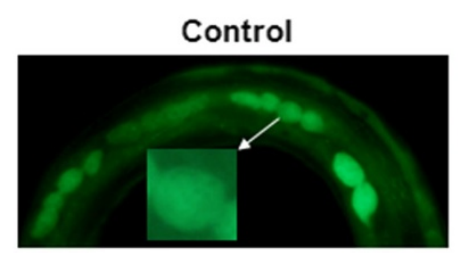

B

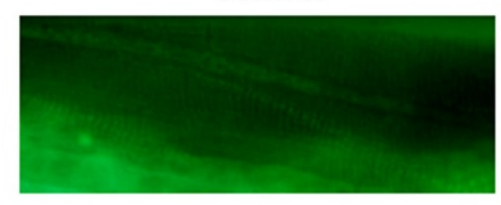

C
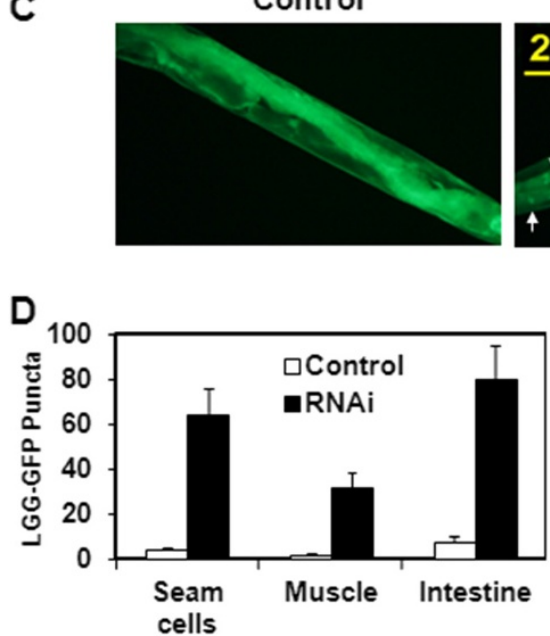

ceMTM3 RNAi

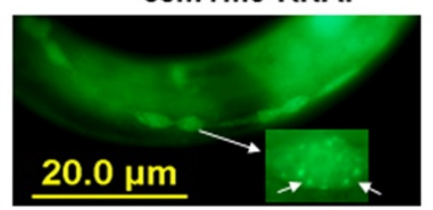

CeMTM3 RNAi

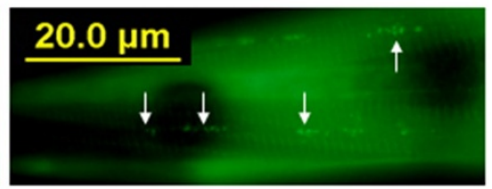

ceMTM3 RNAi

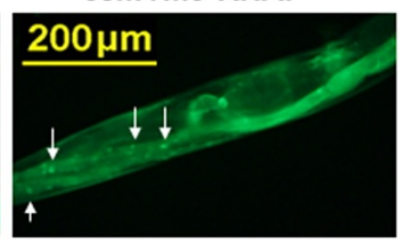

E

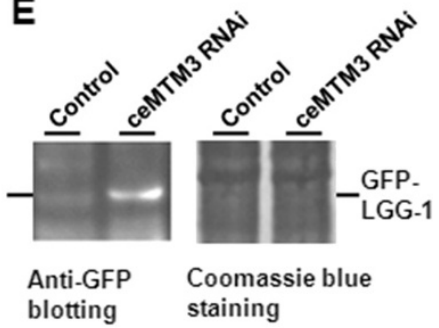

Figure 3 Knockdown of ceMTM3 causes autophagy in multiples tissues of C. elegans worms at different ages. GFP: LGG-1 worms were cultured on NGM plates containing E. coli cells carrying vector control or ceMTM3 RNAi from the time they were hatched from eggs. GFP-positive puncta (indicated by arrows) represent autophagosomes which are abundant in ceMTM3 RNAi-treated worms. A. Autophagosomes in seam cells of day 2 worm larvae. B. Autophagosomes in muscle cells of day 3 young adult worms. Note that muscle fiber bands are visible in the background. C. Autophagosomes in intestine on day 5 adult worms. D. Average numbers of GFP puncta in 4 seam cells, 60 micrometer-long muscles, and entire intestine. Error bars denote standard deviation, $n \geq 20, p<0.001)$. E. Western blotting analyses of lipid-bound GFP-LGG-1. Control and ceMTM3 RNAi-treated worms (mixed ages) were extracted in phosphate-buffer saline by sonication. After centrifugation at $800 \mathrm{~g}$ for $10 \mathrm{~min}$, the supernatants were collected and subjected to further centrifugation at 100,000 for $1 \mathrm{~h}$. The pellets were extracted in SDS gel sample buffer and directly used for western blotting analysis with an anti-GFP antibody. The position of GFP-LGG-1 is indicated. Equal protein loading is demonstrated by Coomassie blue staining.

outer nuclear membrane and in the endoplasmic reticulum [20,21]. Our current study demonstrated that knockdown of ceMTM3, a PI3P phosphatase predominately expressed in the muscle, results in autophagy which subsequently leads to loss of muscle fibers. In a sense, one may postulate that inactivation of ceMTM3 is equivalent to activation of VPS34 in regulating the autophagy process. Therefore, PI3P phosphatases and PI3 kinases play equally important roles in regulation of autophagy.

The role of autophagy in muscle wasting and maintenance has been well documented [14]. Autophagy appears to be a double edged sword. Too much or too little of it can cause muscle weakness and atrophy. For example, excessive autophagy, induced by denervation and fasting, contributes to muscle atrophy [22]. By demonstrating that occurrence of autophagy upon knockdown of ceMTM3 precedes muscle fiber loss, our study provides further proof of the harmful effects of too much autophagy in muscle function. On the other hand, deficiency in autophagy is also known to cause muscle atrophy. For example, both $\operatorname{Atg} 5^{-1-}$ and $\operatorname{Atg} 7^{-1-}$ mice show muscle loss accompanied by accumulation of protein aggregates and abnormal membranous structures in the muscle cells $[23,24]$. By the same concept, our study demonstrated that autophagy-deficient atg-18 worms exhibited impaired body movement and loss of muscle fibers (Figure 6). In addition, an earlier study demonstrated 


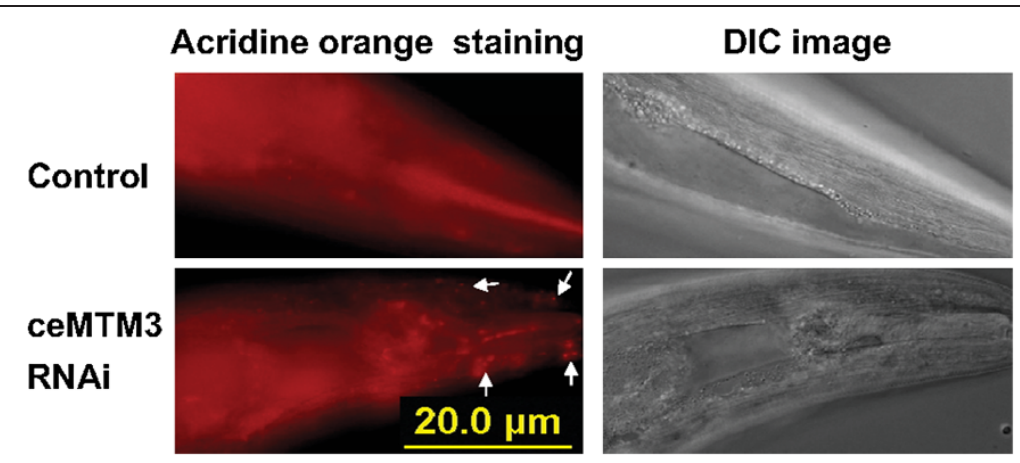

Figure 4 Knockdown of ceMTM3 induces increased lysosomal activity. Day 5 adult worms were treated with acridine orange on NGM plates. Data represent fluorescence and DIC images of the same worm sections. Arrows indicate lysosomes which are abundant in ceMTM3 RNAi-treated worms. Note that muscle fibers are visible in DIC images, indicating increased numbers of lysosomes are present in muscle cells.

that loss of autophagy function due to inactivation of unc-51/atg1 and bec-1/atg6/beclin1 results in small body size without affecting cell number [25]. Our current data demonstrated that excessive autophagy associated with knockdown of ceMTM3 also causes shortening of body size with smaller muscle cells. Therefore, autophagy appears to be a dynamic process and should be kept in a balance. From a therapeutic perspective, it is important to understand whether the reduction of autophagy is helpful during muscle loss or whether enhancement of autophagosome flux is beneficial for the clearance of dangerous organelles or toxic proteins. To keep healthy muscle function, we need to keep a normal level of autophagy flux to rejuvenate organelles and to remove dysfunctional mitochondria and ER membranes but to avoid excessive autophagy which may lead to breakdown of normal muscle fibers.
The loss of muscle mass, referred to as sarcopenia, is a normal phenomenon in animals as a consequence of aging [26]. For humans, decrease in muscle tissue begins around the age of 50 years and becomes more dramatic beyond the 60th year of life. For C. elegans worms, loss of muscle fibers and consequent impairment of body movement is clearly seen after day 15 and is much accelerated by knockdown of ceMTM3 (Figure 1). Aging is associated with a progressive decline of muscle mass, strength, and quality, but the mechanism underlying the muscle wasting remains unresolved [27]. Studies have shown that sarcopenia is not due to lack of regenerative drive in senescent skeletal muscle [28], implying that the maintenance of existing muscle is crucial. Among the many factors correlating with sarcopenia during aging, oxidative stress has been extensively investigated [29]. We believe that oxidation stress-induced inactivation of

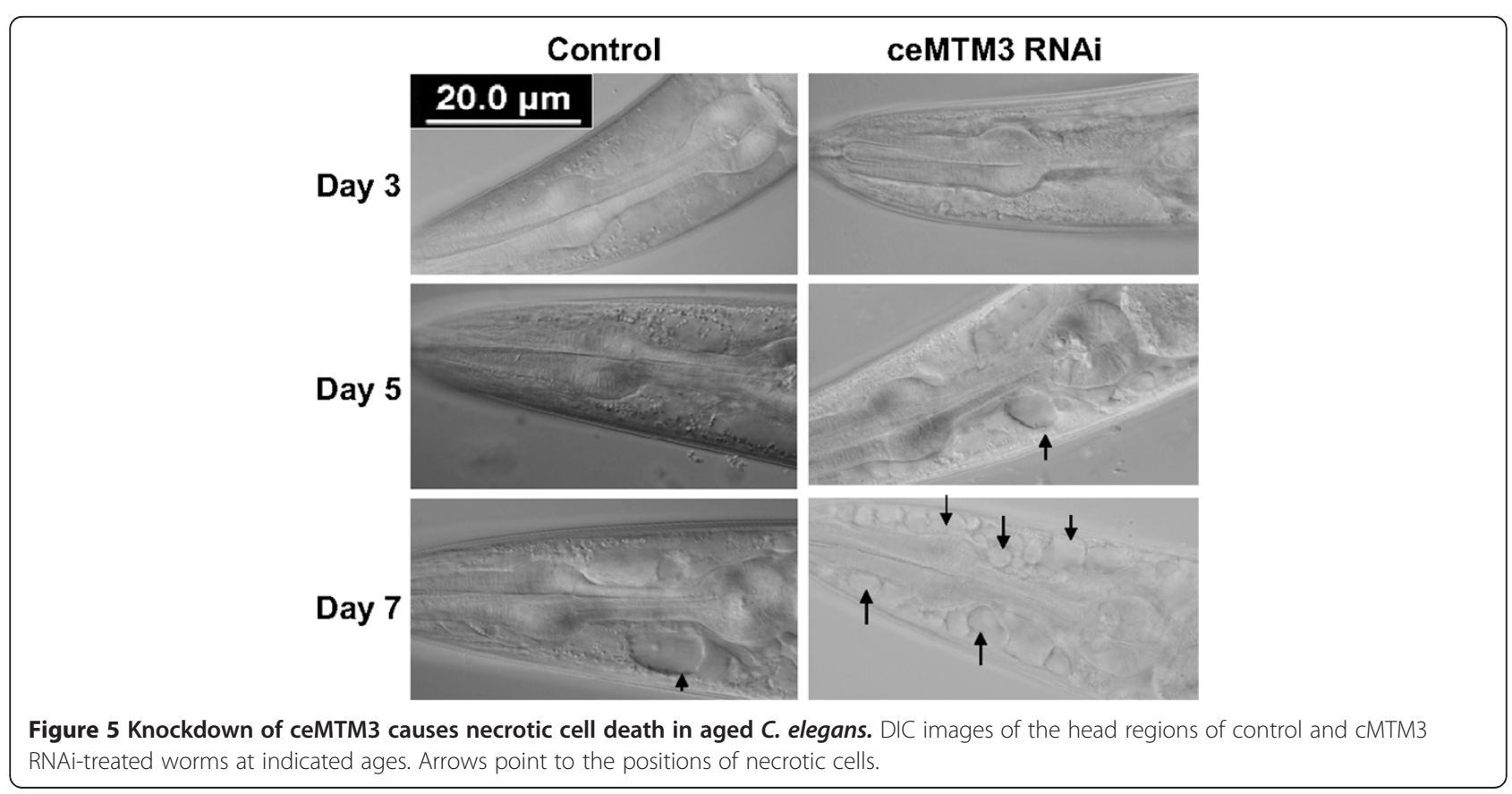




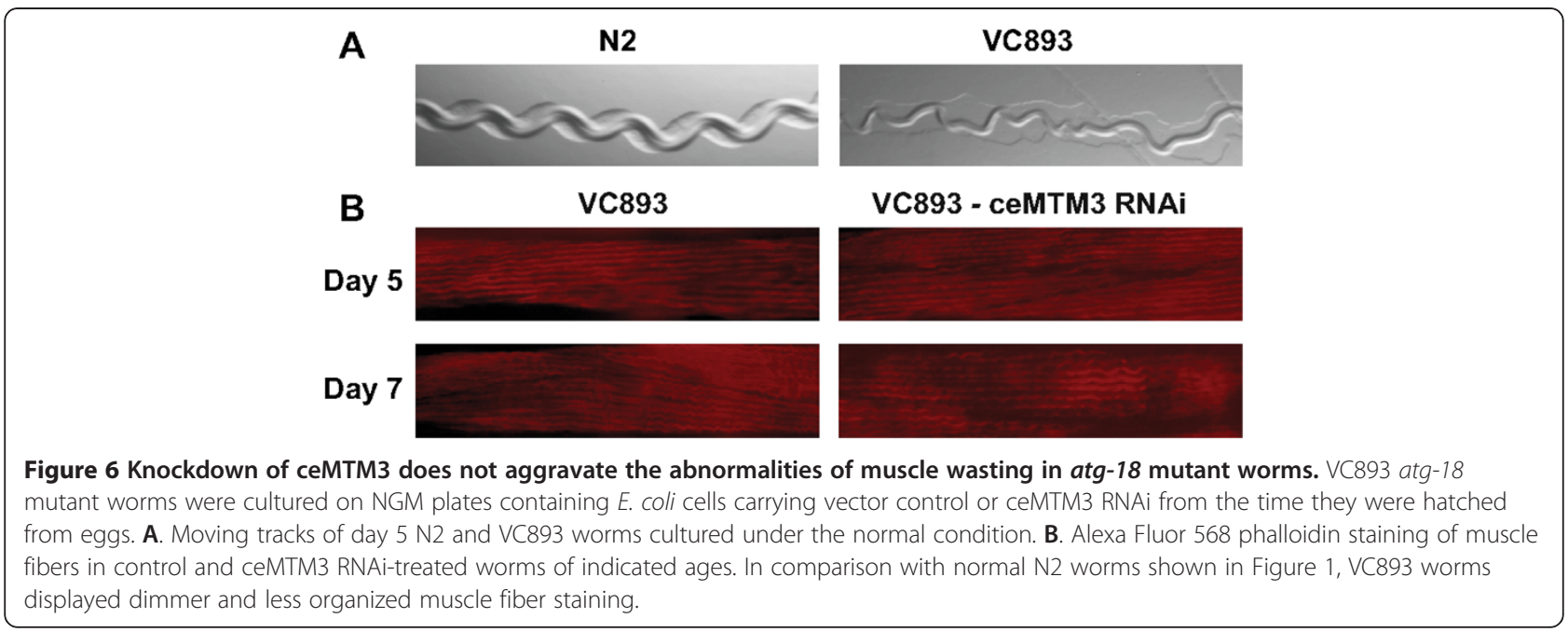

MTM family phosphatases may play a major role in the muscle wasting process. Like all the other members of the tyrosine phosphatase superfamily, MTM family enzymes are susceptible to oxidation-induced inactivation because they contain a highly reactive cysteinyl residue at the catalytic center. Reactive oxygen species, such as the superoxide radical $\left(\mathrm{O}_{2}^{-*}\right)$, hydrogen peroxide $\left(\mathrm{H}_{2} \mathrm{O}_{2}\right)$, the hydroxyl radical $\left(\mathrm{OH}^{*}\right)$, and nitric oxide (NO) are produced in muscle at rest, and this generation is increased by contractile activity [30]. Oxidative damage is considered the main cause of aging. In fact, it was thought that a slower accumulation of oxidative damage is at least partly responsible for the life extension effects seen in C. elegans with daf-2 and age-1 mutations [31]. Our earlier data have shown that ceMTM3 is sensitive to peroxide and loss of ceMTM3 function causes muscle deterioration in C. elegans [12]. Therefore, inactivation of ceMTM3 by oxidation may be attributable to loss of muscle fibers in normal worms at later ages. Thus, to prevent sarcopenia, it is important to maintain a normal level of PI3P phosphatase activity.

\section{Conclusion}

By using the C. elegans worm as a model system, our study provides further evidence that the MTM family phosphatases play a negative role in autophagy. Our study also demonstrates a correlation between excessive autophagy and muscle wasting, implying a normal level of autophagy is important for muscle function and maintenance. Considering sensitivity of tyrosine phosphatase superfamily enzymes to reactive oxygen species, loss of muscle fibers during normal aging may be a consequence of PI3P phosphatase inactivation. Therefore, studying the MTM enzymes may have clinical implications in the prevention and treatment of human sarcopenia.

\section{Methods}

Nematode strains and maintenance

C. elegans worms were grown on nematode growth medium (NGM) plates with abundant $E$. coli food at $20^{\circ} \mathrm{C}$ according to standard protocols. Wild-type Bristol N2, ATG-18-deficient strain VC893 [atg-18(gk378) V], and GFP::myosin heavy chain A-expressing RW1596 [myo-3(st386) V; stEx30] worms were obtained from the Caenorhabditis Genetics Center. Transgenic N2 worms [Plgg-1::gfp::lgg-1+rol-6] carrying the fusion protein GFP::LGG-1 autophagy marker were kindly provided by Dr. Beth Levine (University of Texas Southwestern Medical School) [15]. All worms were scored at the same chronological age and were moved to new plates every day after they reached the reproductive period to avoid progeny contamination. Day 0 refers to the laid egg stage.

\section{Knockdown of ceMTM3}

The full-length coding sequence of ceMTM3 ( -6 to 2,910 with translation starting codon ATG starting from 1) was cloned into the pPD129.36 vector. Plain p129.36 vector was used as control throughout the study. The HT115 (DE3) E. coli cells were employed as hosts for expression of double-stranded RNAs, and 0.4 mM IPTG was used to induce expression of dsRNA. The efficiency of RNAi-medicated knockdown was confirmed by western blotting with anti-ceMTM3 antibodies as previously described [12].

\section{Staining of worms and microscopy}

For phalloidin staining of actin filaments, worms were fixed with cold acetone and then stained with Alexa Fluor 568-phalloidin. For acridine orange staining, $500 \mathrm{ul}$ of $0.01 \mathrm{mg} / \mathrm{ml}$ acridine orange made in M9 buffer was added directly onto worms cultured in a $6 \mathrm{~cm}$ NGM plate to ensure the entire plates were evenly covered. 
After incubation at $20^{\circ} \mathrm{C}$ in the dark for $1 \mathrm{~h}$, the worms were transferred to a new culture plate without acridine orange and allowed to recover at $20^{\circ} \mathrm{C}$ for $1 \mathrm{~h}$ before being immobilized with sodium azide for microscopic analysis [16]. For visualization of fluorescence in GFPpositive worms, worms were treated with sodium azide and then viewed under a fluorescent microscope with a GFP filter. All microscopic analysis was performed with an Olympus BX51 microscope equipped with DIC lens. Digital images were captured using an Olympus DP71 camera with the DP-BSW application software (version 03.02). Quantification of fluorescent signals was performed by using the FluorChem SP program from Alpha Innotech.

\section{Statistical analysis}

Statistical comparison between control and treatment groups was performed with unpaired $t$ test using the GraphPad Prism software (Graphpad Software, La Jolla, CA). Differences with $\mathrm{p}<0.05$ were defined significant.

\section{Abbreviations}

GFP: Green fluorescent protein; NGM: Nematode growth medium; PI3P: Phosphatidylinositol 3-phosphate; RNAi: RNA interference.

\section{Competing interests}

The authors declare no conflict of interests.

\section{Authors' contributions}

$X Y, J M, F L$, and WZ performed the research; XF designed the research; ZJZ designed and supervised the research. All authors wrote, read, and approved the manuscript.

\section{Acknowledgements}

This work was supported by grants HL076309 and HL079441 from the National Institutes of Health (USA) and a pilot grant from the Reynolds Oklahoma Center on Aging (to ZJ Zhao) and by the Doctoral Fund of Ministry of Education of China (No. 20090061110019, to X Fu).

\section{Author details}

'Department of Pathology, University of Oklahoma Health Sciences Center, Oklahoma City, Oklahoma 73104, USA. ${ }^{2}$ Edmond H. Fischer Signal Transduction Laboratory, College of Life Sciences, Jilin University, Changchun 130023, China.

Received: 14 December 2011 Accepted: 17 October 2012

Published: 31 October 2012

\section{References}

1. Xie Z, Klionsky DJ: Autophagosome formation: core machinery and adaptations. Nat Cell Biol 2007, 9:1102-1109.

2. Levine B, Kroemer G: Autophagy in the Pathogenesis of Disease. Cell 2008, 132:27-42.

3. Kourtis N, Tavernarakis N: Autophagy and cell death in model organisms. Cell Death Differ 2009, 16:21-30.

4. Yoshimori T, Noda T: Toward unraveling membrane biogenesis in mammalian autophagy. Curr Opin Cell Biol 2008, 20:401-407.

5. Noda T, Matsunaga K, Taguchi-Atarashi N, Yoshimori T: Regulation of membrane biogenesis in autophagy via PI3P dynamics. Semin Cell Dev Biol 2010, 21:671-676.

6. Robinson FL, Dixon JE: Myotubularin phosphatases: policing 3phosphoinositides. Trends Cell Biol 2006, 16:403-412.

7. Vergne I, Roberts E, Elmaoued RA, Tosch V, Delgado MA, Proikas-Cezanne T, Laporte J, Deretic V: Control of autophagy initiation by phosphoinositide 3-phosphatase Jumpy. EMBO J 2009, 28:2244-2258.
8. Taguchi-Atarashi N, Hamasaki M, Matsunaga K, Omori H, Ktistakis NT, Yoshimori T, Noda T: Modulation of local Ptdlns3P levels by the PI phosphatase MTMR3 regulates constitutive autophagy. Traffic 2010, 11:468-478.

9. Laporte J, Hu LJ, Kretz C, Mandel JL, Kioschis P, Coy J, Klauck SM, Poustka A, Dahl N: A gene mutated in X-linked myotubular myopathy defines a new putative tyrosine phosphatase family conserved in yeast. Nat Genet 1996, 13:175-182.

10. Bolino A, Muglia M, Conforti FL, LeGuern E, Salih MA, Georgiou DM, Christodoulou K, Hausmanowa-Petrusewicz I, Mandich P, Schenone A, Gambardella A, Bono F, Quattrone A, Devoto M, Monaco AP: CharcotMarie-Tooth type $4 \mathrm{~B}$ is caused by mutations in the gene encoding myotubularin-related protein-2. Nat Genet 2000, 25:17-19.

11. Senderek J, Bergmann C, Weber S, Ketelsen UP, Schorle H, Rudnik-Schoneborn S, Buttner R, Buchheim E, Zerres K: Mutation of the SBF2 gene, encoding a novel member of the myotubularin family, in Charcot-Marie-Tooth neuropathy type 4B2/11p15. Hum Mol Genet 2003, 12:349-356.

12. Ma J, Zeng F, Ho WT, Teng L, Li Q, Fu X, Zhao ZJ: Characterization and functional studies of a FYVE domain-containing phosphatase in C. elegans. J Cell Biochem 2008, 104:1843-1852.

13. Meissner B, Warner A, Wong K, Dube N, Lorch A, McKay SJ, Khattra J, Rogalski T, Somasiri A, Chaudhry I, Fox RM, 3rd Miller DM, Baillie DL, Holt RA, Jones SJ, Marra MA, Moerman DG: An integrated strategy to study muscle development and myofilament structure in Caenorhabditis elegans. PLOS Genet 2009, 5:e1000537.

14. Sandri M: Autophagy in health and disease. 3. Involvement of autophagy in muscle atrophy. Am J Physiol Cell Physiol 2010, 298:C1291-C1297.

15. Melendez A, Talloczy Z, Seaman M, Eskelinen EL, Hall DH, Levine B: Autophagy genes are essential for dauer development and life-span extension in C. elegans. Science 2003, 301:1387-1391.

16. Katz DJ, Edwards TM, Reinke V, Kelly WG: A C. elegans LSD1 demethylase contributes to germline immortality by reprogramming epigenetic memory. Cell 2009, 137:308-320.

17. Raben $N$, Shea L, Hill V, Plotz P: Monitoring autophagy in lysosomal storage disorders. Methods Enzymol 2009, 453:417-449.

18. Samara C, Syntichaki $P$, Tavernarakis N: Autophagy is required for necrotic cell death in Caenorhabditis elegans. Cell Death Differ 2008, 15:105-112.

19. Lu Q, Yang P, Huang X, Hu W, Guo B, Wu F, Lin L, Kovács AL, Yu L, Zhang $\mathrm{H}$ : The WD40 repeat Ptdlns(3)P-binding protein EPG-6 regulates progression of omegasomes to autophagosomes. Dev Cell 2011, 21:343-357.

20. Takacs-Vellai K, Vellai T, Puoti A, Passannante M, Wicky C, Streit A, Kovacs AL, Müller F: Inactivation of the autophagy gene bec-1 triggers apoptotic cell death in C. elegans. Curr Biol 2005, 15:1513-1517.

21. Roggo L, Bernard V, Kovacs AL, Rose AM, Savoy F, Zetka M, Wymann MP, Müller F: Membrane transport in Caenorhabditis elegans: an essential role for VPS34 at the nuclear membrane. EMBO J 2002, 21:1673-1683.

22. Wang X, Blagden C, Fan J, Nowak SJ, Taniuchi I, Littman DR, Burden SJ: Runx1 prevents wasting, myofibrillar disorganization, and autophagy of skeletal muscle. Genes Dev 2005, 19:1715-1722.

23. Raben N, Hill V, Shea L, Takikita S, Baum R, Mizushima N, Ralston E, Plotz P: Suppression of autophagy in skeletal muscle uncovers the accumulation of ubiquitinated proteins and their potential role in muscle damage in Pompe disease. Hum Mol Genet 2008, 17:3897-3908.

24. Masiero E, Agatea L, Mammucari C, Blaauw B, Loro E, Komatsu M, Metzger D, Reggiani C, Schiaffino S, Sandri M: Autophagy is required to maintain muscle mass. Cell Metab 2009, 10:507-515.

25. Aladzsity I, Tóth ML, Sigmond T, Szabó E, Bicsák B, Barna J, Regos A, Orosz L, Kovács AL, Vellai T: Autophagy genes unc-51 and bec- 1 are required for normal cell size in Caenorhabditis elegans. Genetics 2007, 177:655-660.

26. Deschenes MR: Effects of aging on muscle fibre type and size. Sports Med 2004, 34:809-824.

27. Thompson LV: Age-related muscle dysfunction. Exp Gerontol 2009, 44:106-111.

28. Edström E, Ulfhake B: Sarcopenia is not due to lack of regenerative drive in senescent skeletal muscle. Aging Cell 2005, 4:65-77.

29. Rossi P, Marzani B, Giardina S, Negro M, Marzatico F: Human skeletal muscle aging and the oxidative system: cellular events. Curr Aging Sci 2008, 1:182-191. 
30. Jackson MJ, McArdle A: Age-related changes in skeletal muscle reactive oxygen species generation and adaptive responses to reactive oxygen species. J Physiol 2011, 589:2139-2145.

31. Honda $Y$, Honda S: Oxidative stress and life span determination in the nematode Caenorhabditis elegans. Ann N Y Acad Sci 2002, 959:466-474.

doi:10.1186/1471-2121-13-28

Cite this article as: Yu et al:: Myotubularin family phosphatase ceMTM3

is required for muscle maintenance by preventing excessive autophagy in Caenorhabditis elegans. BMC Cell Biology 2012 13:28.

\section{Submit your next manuscript to BioMed Central and take full advantage of:}

- Convenient online submission

- Thorough peer review

- No space constraints or color figure charges

- Immediate publication on acceptance

- Inclusion in PubMed, CAS, Scopus and Google Scholar

- Research which is freely available for redistribution 